\title{
Photometric redshifts as a tool for studying the Coma cluster galaxy populations ${ }^{\star}$
}

\author{
C. Adami ${ }^{1}$, O. Ilbert ${ }^{1,2}$, R. Pelló ${ }^{3}$, J. C. Cuillandre ${ }^{4}$, F. Durret ${ }^{5}$, A. Mazure ${ }^{1}$, J. P. Picat ${ }^{3}$, and M. P. Ulmer ${ }^{1,6}$ \\ ${ }^{1}$ LAM, OAMP, Université Aix-Marseille \& CNRS, Pôle de l'Étoile, Site de Château Gombert, 38 rue Frédéric Joliot-Curie, \\ 13388 Marseille 13 Cedex, France \\ e-mail: christophe.adami@oamp.fr \\ 2 Institute for Astronomy, 2680 Woodlawn Dr., University of Hawaii, Honolulu, Hawaii 96822, USA \\ 3 Laboratoire d'Astrophysique de Toulouse-Tarbes, Université de Toulouse, CNRS, 14 Av. Édouard Belin, 31400 Toulouse, France \\ 4 Canada-France-Hawaii Telescope Corporation, Kamuela, HI 96743, USA \\ 5 Institut d'Astrophysique de Paris, CNRS, UMR 7095, Université Pierre et Marie Curie, 98bis Bd Arago, 75014 Paris, France \\ ${ }^{6}$ Department Physics \& Astronomy, Northwestern University, Evanston, IL 60208-2900, USA
}

Received 26 March 2008 / Accepted 13 September 2008

\section{ABSTRACT}

\begin{abstract}
Aims. We apply photometric redshift techniques to an investigation of the Coma cluster galaxy luminosity function (GLF) at faint magnitudes, in particular in the $u^{*}$ band where basically no studies are presently available at these magnitudes.

Methods. Cluster members were selected based on probability distribution function from photometric redshift calculations applied to deep $u^{*}, B, V, R, I$ images covering a region of almost $1 \mathrm{deg}^{2}$ (completeness limit $R \sim 24$ ). In the area covered only by the $u^{*}$ image, the GLF was also derived after a statistical background subtraction.

Results. Global and local GLFs in the $B, V, R$, and $I$ bands obtained with photometric redshift selection are consistent with our previous results based on a statistical background subtraction.

The GLF in the $u^{*}$ band shows an increase in the faint end slope towards the outer regions of the cluster.

The analysis of the multicolor type spatial distribution reveals that late type galaxies are distributed in clumps in the cluster outskirts, where X-ray substructures are also detected and where the GLF in the $u^{*}$ band is steeper.

Conclusions. We can reproduce the GLFs computed with classical statistical subtraction methods by applying a photometric redshift technique. The $u^{*}$ GLF slope is steeper in the cluster outskirts, varying from $\alpha \sim-1$ in the cluster center to $\alpha \sim-2$ in the cluster periphery. The concentrations of faint late type galaxies in the cluster outskirts could explain these very steep slopes, assuming a short burst of star formation in these galaxies when entering the cluster.
\end{abstract}

Key words. galaxies: clusters: individual: Coma - galaxies: luminosity function, mass function

\section{Introduction}

Ever-growing optical imaging surveys of galaxies for which complete spectroscopic follow-up is impossible due to telescope limitations has triggered the development of photometric redshift techniques (e.g. Bolzonella et al. 2000; or Ilbert et al. 2006a, and references therein). Based on the comparison of multi-band photometry with synthetic spectral templates, this technique can be viewed as very low-resolution spectroscopy. The quality of photometric redshifts depends on the wavelength range covered by the photometric survey. High-quality photometric redshifts and related quantities require a complete and

\footnotetext{
* Based on observations obtained with MegaPrime/MegaCam, a joint project of CFHT and CEA/DAPNIA, at the Canada-France-Hawaii Telescope (CFHT) which is operated by the National Research Council (NRC) of Canada, the Institut National des Sciences de l'Univers of the Centre National de la Recherche Scientifique (CNRS) of France, and the University of Hawaii. This work is also partly based on data products produced at TERAPIX and the Canadian Astronomy Data Centre as part of the Canada-France-Hawaii Telescope Legacy Survey, a collaborative project of NRC and CNRS. Also based on data from W. M. Keck Observatory which is operated as a scientific partnership between the California Institute of Technology, the University of California, and NASA. It was made possible by the generous financial support of the W. M. Keck Foundation.
}

contiguous coverage of the spectral regions of interest, in particular around the strong spectral features, such as the $4000 \AA$ break or the Lyman $\alpha$ break. This technique has proven to be a very valuable tool for several cosmological purposes, such as deriving field galaxy correlation functions or galaxy luminosity functions (see e.g. Ilbert et al. 2006b; Meneux et al. 2006). It has also been applied in the study of distant clusters of galaxies (see e.g. White et al. 2005, and references therein).

In the present paper, we apply the photometric redshift technique to study the galaxy population of the rich Coma cluster. Due to its proximity $(z \sim 0.023)$, Coma covers a large extent over the sky (on the order of $1 \mathrm{deg}^{2}$ ), requiring a wide field camera; data in the $U$ band are needed to determine with a sufficient accuracy the probability that a galaxy belongs or not to the cluster, based in particular on the $4000 \AA$ A break.

As a continuation of our Coma photometric survey (e.g. Adami et al. 2006a) which already includes deep $(R \sim 24)$ wide field $\left(42 \times 52 \operatorname{arcmin}^{2}\right) B, V, R$ and $I$ images, we recently acquired a Megacam $u^{*}$ band image of comparable depth and field of view. In addition to our spectroscopic redshift catalog, this allows us to compute photometric redshifts and Probability Distribution Function (PDF hereafter) along the Coma cluster line of sight down to the dwarf galaxy regime. One of the main goals of the paper is to qualify this photometric redshift 
technique applied to the galaxy luminosity function (GLF hereafter) determination. For this, we will compare the present photometric redshift technique with the galaxy luminosity functions computed with the same data set but with a statistical background removal (Adami et al. 2007a,b).

We will also compute luminosity functions in the $u^{*}$ band based on statistical background subtraction in the area where only the $u^{*}$ band is available.

We describe our new photometric data and photometric redshift and PDF estimates in Sect. 2. We compute in Sect. 3 the Coma GLF using the PDF and in Sect. 4 the Coma $u^{*}$ GLF applying statistical subtraction. The spatial distributions of multicolor galaxy types are discussed in Sect. 5. Conclusions are drawn in Sect. 6.

In this paper we assume $\mathrm{H}_{0}=70 \mathrm{~km} \mathrm{~s}^{-1} \mathrm{Mpc}^{-1}, \Omega_{\mathrm{m}}=0.3$, $\Omega_{\Lambda}=0.7$, a distance to Coma of $100 \mathrm{Mpc}$, a distance modulus $=$ 35.00 , and a scale of $0.46 \mathrm{kpc} \operatorname{arcsec}^{-1}$.

\section{Photometric data and photometric redshifts}

\section{1. $B, V, R$ and I band imaging data}

These data are fully described in Adami et al. (2006a) and we give here only the salient points. Coma was observed with a 2 field mosaic of the CFH12K camera in 4 bands $(B, V, R$ and $I)$. Altogether, our data cover a $52 \times 42 \operatorname{arcmin}^{2}$ field over the sky (the CFH12K f.o.v. in the following) centered on the two dominant cluster galaxies (NGC 4874 and NGC 4889) with a completeness level in $R$ close to $R \sim 24$. The seeing conditions were all close to 1 arcsec. These data are available at http: // cencosw. oamp. fr. We have already published several papers based on these data (Adami et al. 2005a,b, 2006a,b, 2007a,b).

\subsection{New $u^{*}$ band imaging data}

New $u^{*}$ band data (see Fig. 3) including the previous field were obtained between 2006 and 2007 with the CFH Megacam camera. Megacam is a mosaic of 36 individual CCDs, giving a field of view (f.o.v.) of $1 \mathrm{deg}^{2}$. The average seeing was 1.1 arcsec. The total exposure time was $9.66 \mathrm{~h}$, obtained by combining 58 individual spatially dithered exposures of $10 \mathrm{~min}$ each. We show in Fig. 1 the weight map that was generated during the reduction procedure. This gives a good idea of the exposure time variations across the field of view (the darker the color, the shorter the exposure time). The sigma of the distribution of the exposure times per pixel is $\sim 30 \%$ of the mean value. However, this affects only the gaps between individual CCDs, that represent less than $10 \%$ of the total area.

The data reduction was performed with the Terapix tools (http://terapix.iap.fr/) and the standard procedure applied for example to the CFHTLS fields (McCracken et al. 2008). A $\sim 1 \mathrm{deg}^{2}$ image was produced with a zero point set to 30.0. We extracted an object catalog from this image with the SExtractor package (Bertin \& Arnouts 1996) with a detection threshold of 0.4 and a minimum number of pixels (above this threshold) of 3 . We derived total Kron AB magnitudes (Kron 1980) to compute photometric redshifts. We corrected each detected object for galactic extinction using the Schlegel maps. This catalog was then cross-correlated with the $B, V$, $R$, and $I$ catalog already available. The cross-correlation was made by applying a technique similar to that of Adami et al. (2006a) and optimized to take into account small astrometric differences between CFH12K and Megacam data. We defined small regions 2 arcmin wide in which we iteratively adjusted

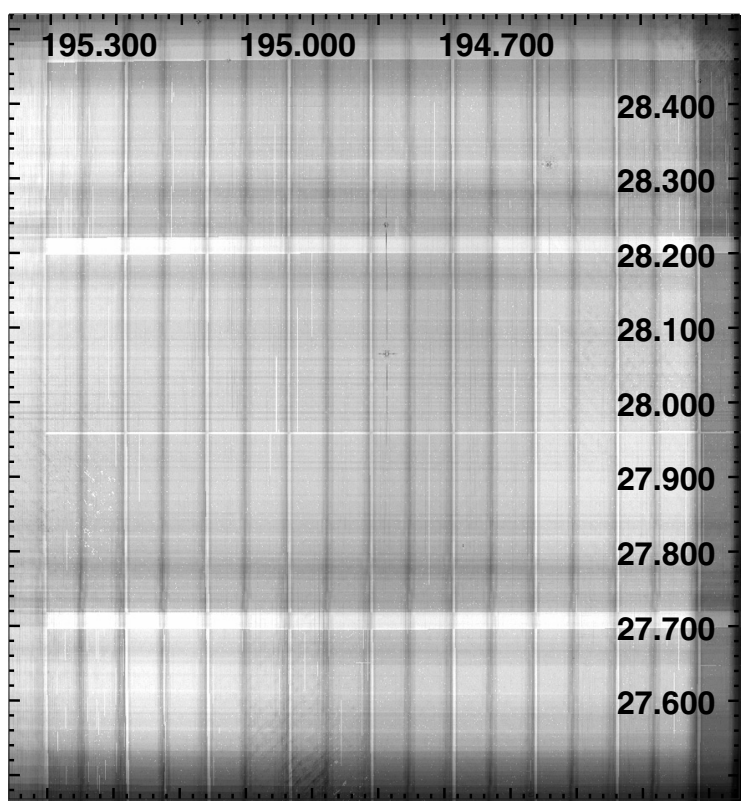

Fig. 1. Megacam $u^{*}$ band weight image (the darker the color, the shorter the exposure time). The coordinates in degrees are indicated.

the galaxy positions (assuming an initial 3 arcsec identification distance) in order to find the best identification. We could also have applied SExtractor in double-image mode, but this would have required to degrade all the Megacam images to the CFH12K characteristics.

We note that the seeing values slightly differed from one band to another, and this could produce offsets in the estimated magnitude values. We did not attempt to correct for this seeing effect before computing the photometric redshifts. However, it was taken into account when fitting the observed magnitudes to the synthethic SEDs by the photometric redshift codes for objects of our spectroscopic catalog (see following). These corrections proved to be small.

\subsection{Zero point variations across the field of view}

Besides the possible seeing effects, it is important to assess possible magnitude zero point variations across our field of view. Given the $u^{*}$ data treatment, the $u^{*}$ zero points are stable over the whole field of view at better than 0.05 mag (a calibration at better than $\sim 4 \%$ is achieved by the Terapix Elixir code from the field center to the field edges). Regarding the CFH12K data, we already estimated in Adami et al. (2006a) that correction in magnitude due to small airmass variations would have been, at maximum, of $0.033 \mathrm{mag}$ for $R, 0.020 \mathrm{mag}$ for $V$, and $0.015 \mathrm{mag}$ for $B$, much less than the estimated errors on the magnitudes. In the same paper (Fig. 16) we also considered stellar tracks to estimate the additional shifts that must be applied to the zero points to match the empirical stellar library of Pickles (1998). The mean (over the whole f.o.v.) corrections were negligible. However, this does not exclude some possible small zero point variations accross the field of view that could remain undetected due to the width of the observed stellar tracks (on the order of $0.2 \mathrm{mag}$ ). In order to test this point, we redid the same figure as Fig. 16 of Adami et al. (2006a), separating the northern and the southern area (as defined in Adami et al. 2007a). Figure 2 shows that we nearly have the same agreement between the empirical stellar library of Pickles (1998) and the observed stellar tracks for both the northern and southern fields. We estimated the mean 

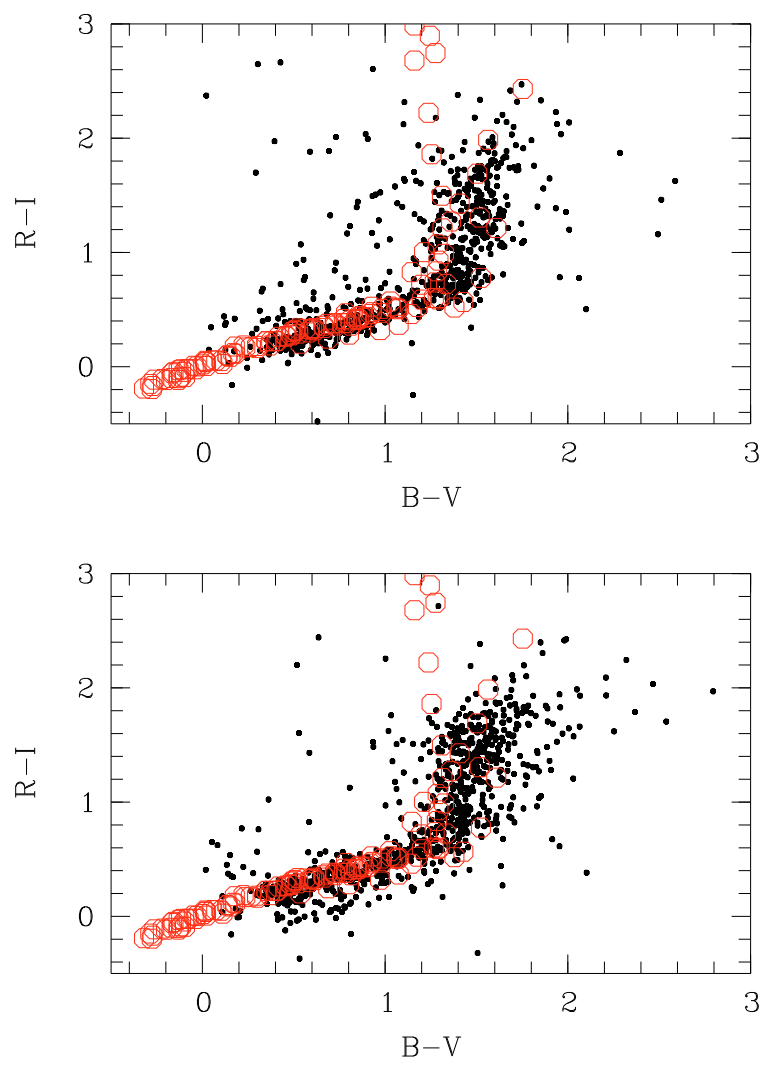

Fig. 2. $R-I$ versus $B-V$ for stars (as defined in Adami et al. (2006a). Dots: observed stars, open red (grey in black and white version) circles: empirical stellar library of Pickles (1998). The upper and lower figures correspond to the north and south CFH12K fields respectively.

magnitude shift between the northern and southern fields to be smaller than 0.05 mag.

\subsection{Completeness of the $u^{*}$ image}

The good efficiency of Megacam in the blue allowed us to reach a similar depth in $u^{*}$ as in the B $(\sim 24.75$ for point like objects at the $90 \%$ level, see Adami et al. 2006a), $V(\sim 24), R(\sim 24)$, and $I$ ( $\sim 23.25)$ images. These new data were required to compute photometric redshifts in order to have a blue photometric band, encompassing the $4000 \AA$ break.

The percentage of $R$ detections with an associated $u^{*}$ detection is between $87 \%$ and $92 \%$. This leads to estimate the $90 \%$ completeness level of the $u^{*}$ image to $R \sim 24$. The $\sim 10 \%$ of galaxies detected in $R$ and not in $u^{*}$ are, at least partially, objects that are not forming stars and which therefore do not appear in $u^{*}$ even in a deep exposure.

In order to assess the completeness level of our $u^{*}$ image in another way, we directly compared our $u^{*}$ counts with the $u^{*}$ counts of the CFHTLS D1, D3 and D4 fields (T0004 release). These fields have long enough exposure times (see http://terapix . iap. fr/cplt/table-syn-T0004 .html) to be comparable with our data. We did not consider the D2 field that only has a total exposure time of $1.3 \mathrm{~h}$ in $u^{*}$. Figure 4 shows that the Coma counts dominate the D1, D3, and D4 counts for $u^{*} \leq 25$, due to the presence of the highly populated Coma cluster in the field. Our data become incomplete at fainter magnitudes, leading to estimate the $u^{*}$ completeness level to $\sim 25.5$. Given that the $u *-R$ color in our data is on the order of 1.5 , this $u^{*}$ completeness level is fully consistent with the previously estimated $R$ completeness level $(R \sim 24)$.

\subsection{Photometric redshift techniques}

We have computed photometric redshifts with two of the most well-known packages: Hyperz (e.g. Bolzonella et al. 2000 and ${ }^{1}$ ) and LePhare (e.g. Ilbert et al. 2006a and ${ }^{2}$ (authors: Arnouts \& Ilbert)), mainly to check that these two softwares give consistent results. These tools are fully described in the quoted papers. In a few words, Hyperz (Bolzonella et al. 2000) and LePhare (Arnouts \& Ilbert) are both based on a template-fitting procedure. However, the setting of the two photo- $z$ codes presents two main differences: Hyperz includes a large set of templates from Bruzual \& Charlot (2003) with different star formation histories and different ages. LePhare includes a limited library of 9 templates from Polletta et al. (2007) and 3 star-forming templates from Bruzual \& Charlot (2003). In Hyperz, the zero-point calibration has been done by comparing the stellar locus in a color-color diagram with the expected star colors. In LePhare, the zero-point calibration has been done with an iterative procedure described in Sect. 4.1 of Ilbert et al. (2006a), by comparing the predicted magnitudes from the best fit template and the observed magnitudes. In order to compute these possible small magnitude zero point shifts in LePhare, we trained the photometric redshift estimates with the spectroscopic catalog described in Adami et al. (2005a). This catalog was supplemented with 20 archive redshifts from the Keck LRIS multispectrograph (Secker et al. 1998), increasing the total number of redshifts available in the CFH12K f.o.v. by $\sim 5 \%$. These galaxies have magnitudes between $R=16.5$ and 21.5 and redshifts lower than 0.5 (only one of these objects is part of the Coma cluster).

The additional zero point shifts applied to our photometry to compute optimal photometric redshifts with Hyperz and LePhare were quite small. Hyperz required manually imposed shifts of less than 0.05 mag in $R$ and $I$ and no shift in $u^{*}, B$ and $V$. LePhare required shifts of $0.028 \pm 0.02$ in $u^{*},-0.141 \pm 0.09$ in $B,-0.062 \pm 0.03$ in $V, 0 . \pm 0.04$ in $R$ and $0.12 \pm 0.05$ in $I$ (error bars coming from the dispersion of the shifts over the whole spectroscopic sample).

A set of fit parameters was then produced (see also Adami et al. 2008): internal extinction, age, multicolor type (based on a color classification in a 5 mag space), redshift, and PDF estimates. However, we mainly consider here the (photometric) redshift, the template multicolor type, and the PDF estimates. Types 1 to 4 are the Elliptical, Sbc, Scd and Irr from Coleman et al. (1980) respectively and types 5 are starburst 1 templates from Kinney et al. (1996).

We also computed the integrated probability for each galaxy to be at a redshift lower than a given value $z_{\text {lim }}(P$ hereafter). This is the area of the PDF enclosed in the considered redshift interval: $\left[0 ., z_{\mathrm{lim}}\right]$.

\subsection{Advantages of the photometric redshift technique}

We could argue that a simple color-magnitude relation (CMR hereafter) could also provide a good way to discriminate between cluster members and non members (Biviano et al. 1996). However, besides the simple fact that five bands appear intuitively better than two to characterize the spectral energy distributions of galaxies, the use of a CMR first requires the

\footnotetext{
1 http://webast.obs-mip. fr/hyperz/

2 http://wwW . oamp.fr/people/arnouts/LE_PHARE.html
} 


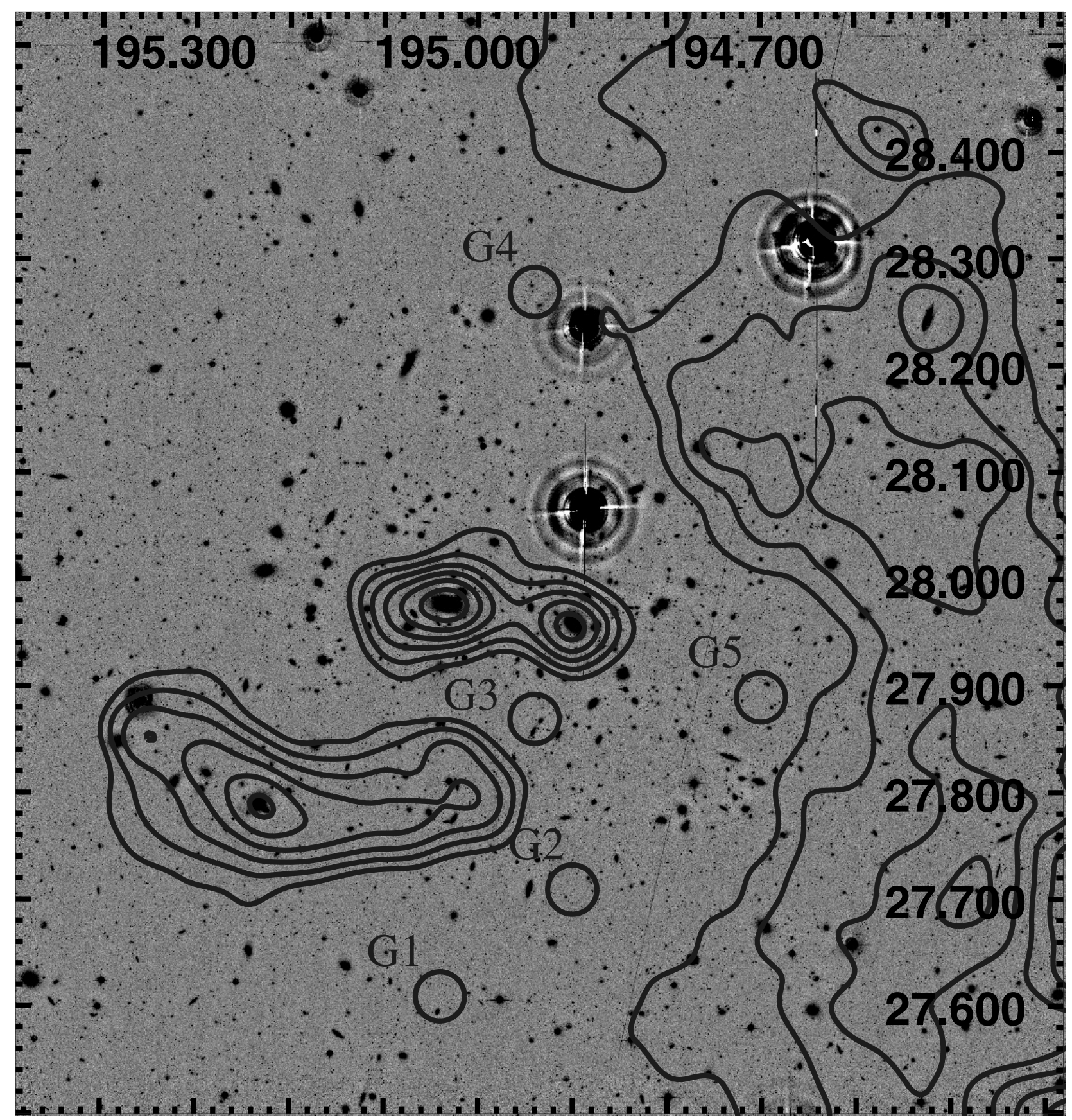

Fig. 3. Megacam $u^{*}$ band image overplotted with the 5 detected groups (circles correspond to $300 \mathrm{kpc}$ in diameter at $z=0.1$ ) and the Neumann et al. (2003) X-ray substructures. We corrected for a small overall shift between the XMM and the Megacam astrometry.

existence of a well defined red sequence (RS hereafter). This is not always verified even if the RS in Coma is very well known at bright magnitudes, thanks to the large percentage of bright elliptical galaxies in this cluster (e.g. Adami et al. 1998). In this case, the photometric redshift and CMR techniques give comparable results.

Second, this RS is only poorly known beyond the present spectroscopic limit (typically $R \sim 21$ for the Coma cluster). It may show a reddening at $R>21$, as suggested by e.g. Adami et al. (2000). Moreover, at $R>21$ peculiar galaxy types appear, directly resulting from the disruptions of larger galaxies. An example are the tidal dwarf galaxies (Bournaud et al. 2003). These peculiar galaxies (not only tidal dwarf galaxies) sometimes have elliptical like spectral types, but are atypically red (e.g. depleted early type galaxy cores shown in Adami et al. 2006b). The simple extrapolation of the bright Coma cluster galaxy $\mathrm{RS}$ at $R>21$ would miss these peculiar objects as cluster members.

Third, the photometric redshift technique offers a much more direct way to characterize the spectral energy distributions of the galaxies. The use of the CMR RS can at best discriminate between early and late type galaxies for not too faint magnitudes. On the other hand, the photometric redshift technique (also see Sect. 2.8) allows the separation of galaxies in 5 spectral types and gives a direct estimate of the ages and internal extinctions of the galaxies.

For all these reasons, we decided to apply the photometric redshift technique in this paper. 


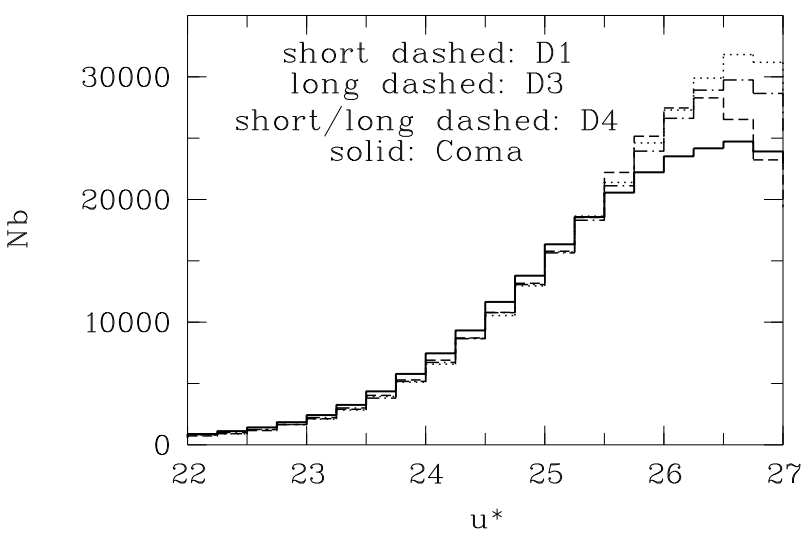

Fig. 4. Coma line of sight $u^{*}$ counts (solid line) compared to three deeper $u^{*}$ fields from the CFHTLS: D1 (short dashed line), D3 (long dashed line), and D4 (short/long dashed line).

\subsection{Results}

First, we limited the catalog to the objects detected in all $u^{*}, B$, $V, R$, and $I$ bands. This excluded the south-west area for which $B$ and $V$ data are not available (see Adami et al. 2006a).

The two sets of estimates (from LePhare and Hyperz) are most of the time in good agreement. As usual, we limited the sample to unmasked regions (this leads to remove all objects at less than twice the radius of objects brighter than $R=18$ ) to avoid uncertain magnitude estimates, and we plotted for both methods the photometric versus spectroscopic redshifts. Our spectroscopic catalog includes 172 spectroscopic redshifts in non masked areas, among which 103 are at $z \leq 0.2$. Figure 5 shows the results for LePhare and Hyperz. As both methods provide very similar results, we merged the two estimates (LePhare and Hyperz) keeping the value with the best reduced $\chi^{2}$ (after having homogenized the two sets of $\chi^{2}$ ).

Beyond the general agreement between photometric and spectroscopic redshifts, we clearly see a degeneracy at the Coma cluster redshift. For some galaxies which have spectroscopic redshifts inside the cluster, LePhare and Hyperz produce photometric redshifts not only at the cluster redshift but spread over the interval $z=0, z \sim 0.2$. Moreover, there is a clear systematic offset on the order of 0.05 in redshift (mainly visible in the LePhare results) between $z=0.1$ and 0.4 . This bias can have two origins. First, the magnitude estimates can be partially biased because of observational effects (small seeing variations between the different photometric bands, peculiar problems for a given band at a given sky location, etc.). Second, cluster galaxies are in general redder than their equivalents in the field due to environmental effects, while photometric redshift estimates are computed with synthetic galaxy templates mainly based on field galaxies. This can lead to a mis-interpretation of the spectral energy distribution, confusing the intrinsic red color and the reddening of galaxies due to redshift. This shows the need for the definition of synthetic cluster galaxy spectroscopic templates. This also implies that we cannot use directly the photometric redshift estimates to efficiently discriminate between cluster members and field galaxies.

Moreover, certain galaxies may have a very extended PDF and considering just the value giving the maximum probability could produce a wrong photometric redshift. We will therefore classify galaxies as being at a greater or lower redshift than a limiting value $z_{\text {lim }}$, based on the probability $P$ quoted above and computed using the area below the PDF for $z$ lower than $z_{\lim }$ for a given galaxy. The value of $z_{\text {lim }}$ will be chosen to minimize the number of intruders (galaxies at spectroscopic redshift greater than $z_{\text {lim }}$ that have a photometric redshift lower than $z_{\mathrm{lim}}$ ) and of lost galaxies (galaxies at spectroscopic redshift lower than $z_{\text {lim }}$ with a photometric redshift greater than $z_{\mathrm{lim}}$ ). This minimization was done on the basis of the spectroscopic catalog and the results are shown in Fig. 6. Setting $z_{\lim }$ to a high value would produce no intruder or lost galaxies, but the discrimination power of such a high value would be low. We therefore selected $z_{\text {lim }}=0.20$, which is a good compromize between intruder and lost galaxy percentages and the discrimination power of the method. We see in Fig. 6 that the percentages of intruder or lost galaxies do not decrease very strongly for $z \geq 0.2$. With this limit and considering our spectroscopic catalog, we estimate that we lose (in the magnitude range covered by the spectroscopic catalog) less than $10 \%$ galaxies and we include about $15 \%$ intruders. These percentages will be taken into account when computing the GLFs in the next section.

\subsection{Uncertainties in the galaxy discrimination based on the PDF}

With this limit of $z=0.2$ and considering our spectroscopic catalog, we estimate that we lose less than $10 \%$ galaxies and we include about $15 \%$ intruder galaxies if we limit our analysis to the spectroscopic catalog magnitude limit.

Given the fact that our spectroscopic catalog is by far not as deep as our photometric catalog, we need to quantify the accuracy of the photometric redshifts beyond the spectroscopic limit. Ilbert et al. (2006a) have shown that the $1 \sigma$ error bars are representative of a measurement at $68 \%$ confidence level, and we can therefore quantify this accuracy based on the $1 \sigma$ error bars. Figure 7 shows the fraction of photometric redshifts from LePhare (results would be similar with Hyperz) with a $1 \sigma$ error bar smaller than $0.2 \times(1+z)(0.2$ being typically the width of the low redshift interval we want to characterize). This figure shows that we still have more than half of the galaxies with a photometric redshift estimate lower than $0.2 \times(1+z)$ for $R$ brighter than 22. We also have a good agreement between the decrease of the fraction of photometric redshifts with a $1 \sigma$ error bar smaller than $0.2 \times(1+z)$ and the number of available spectroscopic redshifts (shown as the histogram in Fig. 7). These percentages will be taken into account when computing the GLF uncertainties in the next section.

\subsection{Uncertainties in the galaxy color-type estimate}

In order to determine the reliability of color-types assigned to galaxies in the Coma cluster, we have carried out a series of simulations with Hyperz and related software. Synthetic cata$\log$ s contain $10^{5}$ galaxies in total at the redshift of Coma, with $R$-band magnitudes ranging between $R=19$ and 24, spanning all the basic spectral types, i.e. $\sim 4000$ galaxies per magnitude and type bin. Photometric errors in the different filters were assigned following a Gaussian distribution with variance scaled to apparent magnitude, i.e. $\sigma(m) \simeq 2.5 \log [1+1 /(S / N)]$, where $S / N$ is the signal to noise ratio corresponding to the apparent magnitude $m$ in our catalog. Hyperz settings used to fit these synthetic catalogs are the same as for real catalogs.

Table 1 summarizes the results obtained from these simulations as a function of $R$-band magnitude and photometric type. Up to $R \leq 23$ (we assumed a limit of $R=22.5$ in the following), photometric types are correctly retrieved by SED fitting, with a 

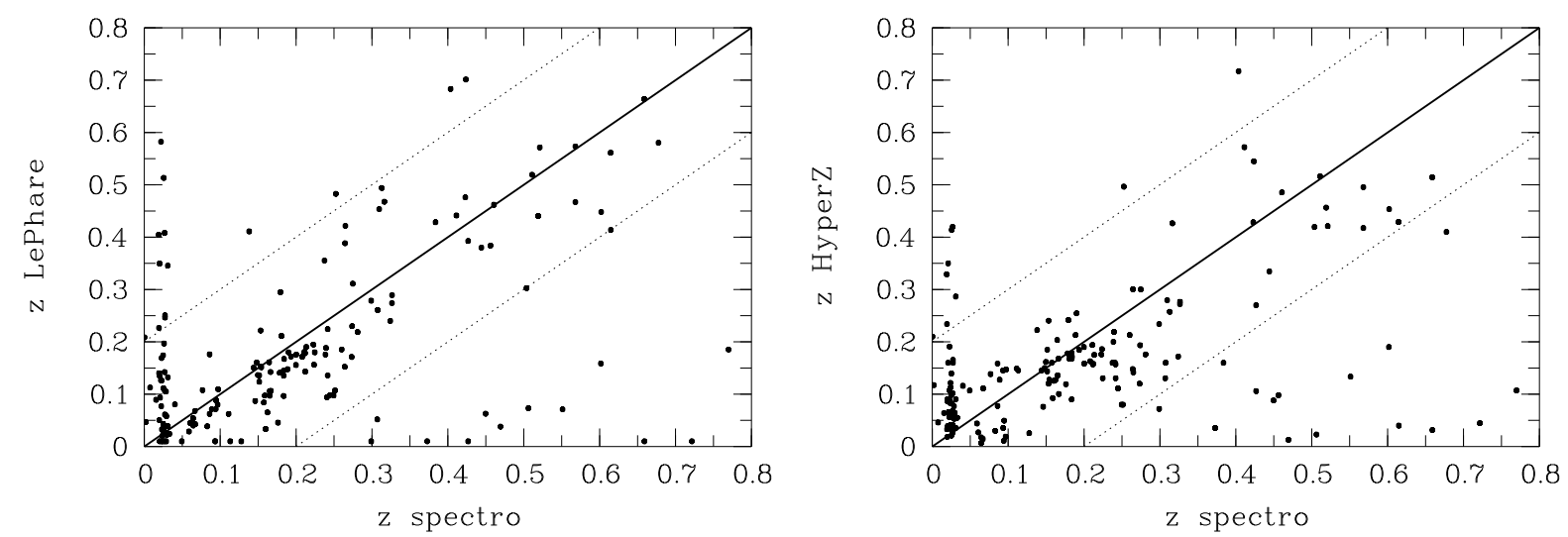

Fig. 5. LePhare (left) and HyperZ (right) photometric redshifts in the $\mathrm{CFH} 12 \mathrm{~K}$ f.o.v. and outside the masked regions versus spectroscopic redshifts. The two dashed lines show a \pm 0.2 uncertainty enveloppe around the perfect relation (continuous line). The large number of dots below $z_{\text {phot }} \sim 0.2$ but outside of Coma is explained by the groups detected in Sect. 3.2.

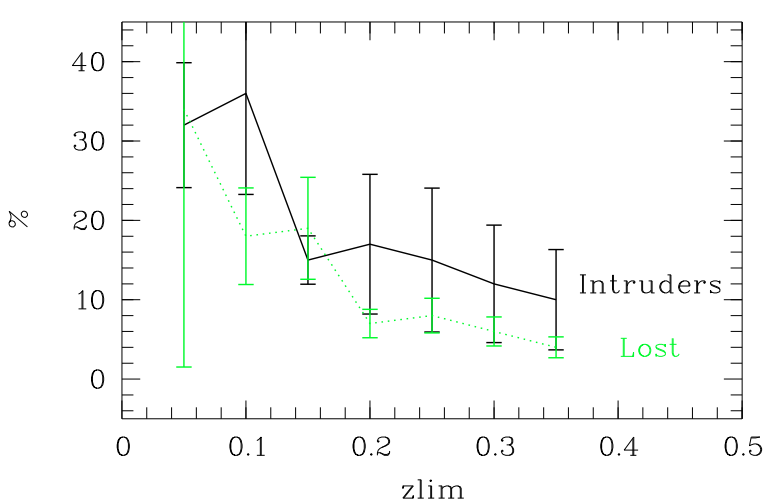

Fig. 6. Variation of the percentage of intruders (full black line) and lost galaxies (green (grey in black and white version) dotted line) as a function of $z_{\text {lim. }}$. Error bars are Poissonian uncertainties.

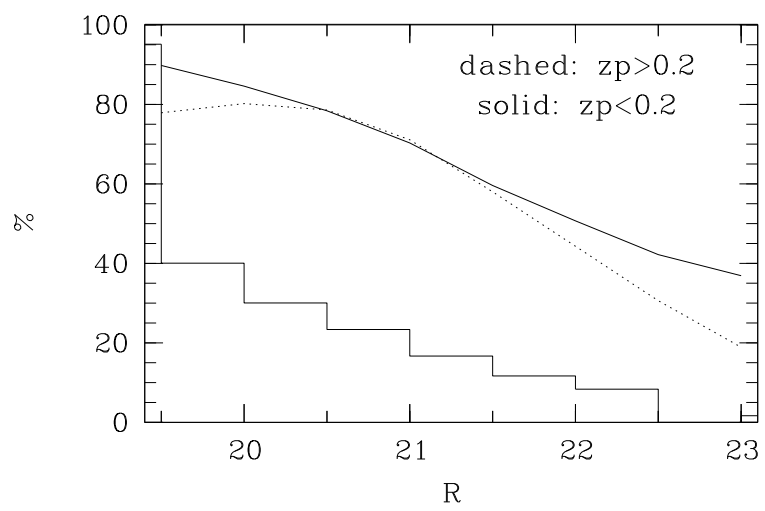

Fig. 7. Fraction of photometric redshifts (as a function of $R$ magnitude) from LePhare with a $1 \sigma$ error bar smaller than $0.2 \times(1+z)$. Solid line: photometric redshifts lower than 0.2 , dashed line: photometric redshifts greater than 0.2. The histogram (in arbitrary units) shows the number of available spectroscopic redshifts as a function of $R$ magnitude.

percentage of failures usually below $\sim 3 \%$, and up to $\sim 10 \%$ for the bluest galaxies. As expected, early-type galaxies are better identified than late types, even for the faintest sources in our catalog, but the difference is small. At least $\sim 75 \%$ of galaxies are still correctly classified in the faintest magnitude bin.

Of course, since we fit the templates used to generate the catalogs, this must help recovering the color-types; the errors
Table 1. Percentage of simulated galaxies in the Coma cluster with photometric types correctly assigned as a function of $R$-band magnitude.

\begin{tabular}{cccccc}
\hline \hline$R$ & E/S0 & Sbc & Scd & Im & SB \\
mag & $\%$ & $\%$ & $\%$ & $\%$ & $\%$ \\
\hline $19-20$ & 100.0 & 99.9 & 99.9 & 98.9 & 98.8 \\
$20-21$ & 100.0 & 99.9 & 99.9 & 98.7 & 99.2 \\
$21-22$ & 100.0 & 99.7 & 99.8 & 97.9 & 97.5 \\
$22-23$ & 100.0 & 98.3 & 98.2 & 93.3 & 90.3 \\
$23-24$ & 98.0 & 88.4 & 84.7 & 76.2 & 77.3 \\
\hline
\end{tabular}

quoted are therefore lower limits on the true uncertainties on the galaxy color-type estimates (see also Sect. 5).

\section{The galaxy luminosity function computation}

As previously stated, photometric redshift techniques allow one to make an optimal subtraction of background galaxies for a redshift limit of 0.2. However, we still need to remove from the sample the galaxies at $z \leq 0.2$ which do not belong to the Coma cluster. These galaxies can be field galaxies or galaxies included in groups of galaxies not related to Coma. We chose to subtract statistically these two contributions by considering field and group luminosity functions taken in the literature. In this way, we are still applying a statistical subtraction, but photometric redshift techniques help to cut down very significantly the redshift range that includes background galaxies.

\subsection{Field contribution}

We estimated the field contribution to be subtracted from the VVDS field luminosity function of Ilbert et al. (2005), who obtained a very large redshift catalog complete to $I(\mathrm{AB}) \sim 24$ (close to $R \sim 24$ considering the passband of our filters). This is close to our own magnitude limit and ensures that the field luminosity function we subtract is really constrained over our whole magnitude range, without requiring any extrapolation.

The Ilbert et al. (2005) luminosity function is also computed in five bands from $U$ to $I$, allowing a field subtraction adapted to each photometric band.

The subtraction of the field contribution was simply done by computing the cosmological co-moving volume included in our field of view at redshift lower than 0.2 and using the $\phi^{*}, \alpha$, and 
Table 2. Characteristics of the background groups detected by the Serna-Gerbal method, based on spectroscopic redshifts: coordinates, mean redshift and total mass (Adami et al. 2005).

\begin{tabular}{lllll}
\hline \hline Group & $\alpha$ & $\delta$ & Mean $z$ & Mass $\left(M_{\odot}\right)$ \\
\hline G1 & 195.04 & 27.61 & 0.149 & $7.34 \times 10^{12}$ \\
G2 & 194.90 & 27.71 & 0.138 & $5.25 \times 10^{11}$ \\
G3 & 194.92 & 27.87 & 0.144 & $1.25 \times 10^{13}$ \\
G4 & 194.94 & 28.27 & 0.097 & $1.46 \times 10^{11}$ \\
G5 & 194.70 & 27.89 & 0.133 & $4.15 \times 10^{12}$ \\
\hline
\end{tabular}

$M^{*}$ parameters of the Schechter function given by Ilbert et al. (2005).

\subsection{Group contribution}

We first located in our field of view the groups unrelated with the Coma cluster and at redshift lower than 0.2 by applying the Serna-Gerbal method (Serna \& Gerbal 1996) to the redshift catalog (Adami et al. 2005a). Briefly, this method is able to detect dynamically linked galaxies (what we call a group of galaxies) based on the positions, magnitudes and redshifts of the galaxies (see Table 2). This method was already applied by Adami et al. (2005a) to study the Coma cluster, and five background groups at $z \leq 0.2$ were detected.

The group luminosity functions were computed from the SDSS group luminosity function estimates of Zandivarez et al. (2006). Since these authors have data in the five SDSS bands, we transformed their magnitudes to our own system applying the relations given by Fukugita et al. (1995). The number of galaxies brighter than $R=17.75$ (where our spectroscopic catalog is nearly complete) was estimated from our spectroscopic cata$\log$. This allowed us to compute the $\phi^{*}$ normalization of the five detected groups (see Fig. 3).

In order to subtract this group contribution, we assumed that the whole group galaxy contribution was enclosed in a $300 \mathrm{kpc}$ diameter circle, a typical group size.

We note here that we do not know the background group GLF per spectral type and this prevents us from computing a Coma cluster GLF per spectral type with the photometric redshift technique. We will simply study the general multicolor type spatial distribution in Sect. 5.

\subsection{GLF uncertainties}

We took into account several uncertainties on the Coma cluster GLF (computed in 0.5 mag bins, as in Adami et al. 2007a):

- the Poisson noise in each bin;

- the uncertainty on the field and group luminosity functions, computed by generating 1000 field and group luminosity functions with parameters Gaussianly varying inside the error range quoted in the literature;

- the uncertainty due to the photometric redshift computations. As previously shown, the number of galaxies below $z=0.2$ can be overestimated by $15 \%$ or underestimated by $10 \%$ within the magnitude range where the spectroscopic catalog is contributing. For fainter magnitudes, we considered Fig. 7 to infer a value of the overestimation and of the underestimation.

The sum of the three uncertainties is assumed to be the total error and is close to a $3 \sigma$ error. The uncertainty due to the photometric redshift computations is clearly the dominating source of error.

\section{Galaxy luminosity functions (GLF)}

\subsection{Galaxy luminosity functions based on photometric redshifts}

We computed the GLF in the subregions previously considered by Adami et al. (2007a). These $\sim 10^{\prime} \times 10^{\prime}$ regions cover the whole cluster area and represent a good compromize between the spatial resolution and the number of galaxies included in the individual GLFs. Figure 8 shows the GLFs together with their $1 \sigma$ error bars (red symbols) overplotted on the previous GLFs computed by Adami et al. (2007a: black symbols) with statistical background subtractions.

The goal of this section is mainly to compare our results with those of Adami et al. (2007a). As these two studies are based on the same dataset, this is a good way to test the reliability of the two methods (statistical field subtraction and photometric redshift technique). The agreement is generally quite good between the two estimates. A few regions, however, show significant discrepancies: fields 5, 6, 8, 9, 16, and 20 . These fields have different luminosity functions (derived with the two techniques) over more than $15 \%$ of the magnitude interval $R=[19.25,22.5]$, the largest differences occuring for fields 16 and 6. It is interesting to note that among these fields, three contain very bright stars (fields 5, 8, and 9) and one (field 8) the cluster dominant galaxy NGC 4874. These objects have a very extended light halo that possibly affects the photometry (even if they were masked inside twice their radius) and therefore the photometric redshift computations, or the faint object detection in the case of the statistical background removal technique of Adami et al. 2007a). We also know that at least one of these fields (field 9) contains a significant population of very faint and very blue Coma galaxies (the field around NGC 4858/4860, Adami et al. 2007b). These faint blue knots, similar to those discussed by Cortese et al. (2007), are perhaps not well represented in our galaxy synthetic templates; this could produce an incorrect value for photometric redshifts, outside the Coma cluster range.

In order to have a third estimate (beyond Adami et al. 2007a, and the present paper determinations), we also compared our results for one of these possibly biased fields (the NGC 4874 region) with a study dedicated to the computation of the GLF in the Coma cluster center (Secker et al. 1996, based on Keck images). Figure 9 shows that these authors predict galaxy number densities consistent at the $2 \sigma$ level with the photometric redshift technique estimates in the range where these counts are lower than the ones computed in Adami et al. (2007a). So the counts of Adami et al. (2007a) could also be overestimated (despite the great care that was devoted to the statistical field subtraction). It is beyond the scope of this paper to present a full comparison with literature results. This was already done in Adami et al. (2007a: Sect. 7.4).

We also note that we still see the discrimination between the north-northeast and the south-southwest parts of the cluster (Fig. 10) that was detected by Adami et al. (2007a). The north-northeast region is more populated in the faint magnitude regime than the south-southwest regions. However, this trend is not significant when applying the photometric redshift technique. We also see in the southern cluster part a similar turn-over as the one already shown in $R$ in Adami et al. (2007a), but it is poorly significant due to the large error bars. These error bars could only be reduced by using a deeper spectroscopic catalog to better constrain the photometric redshift estimates of the faint galaxies. 


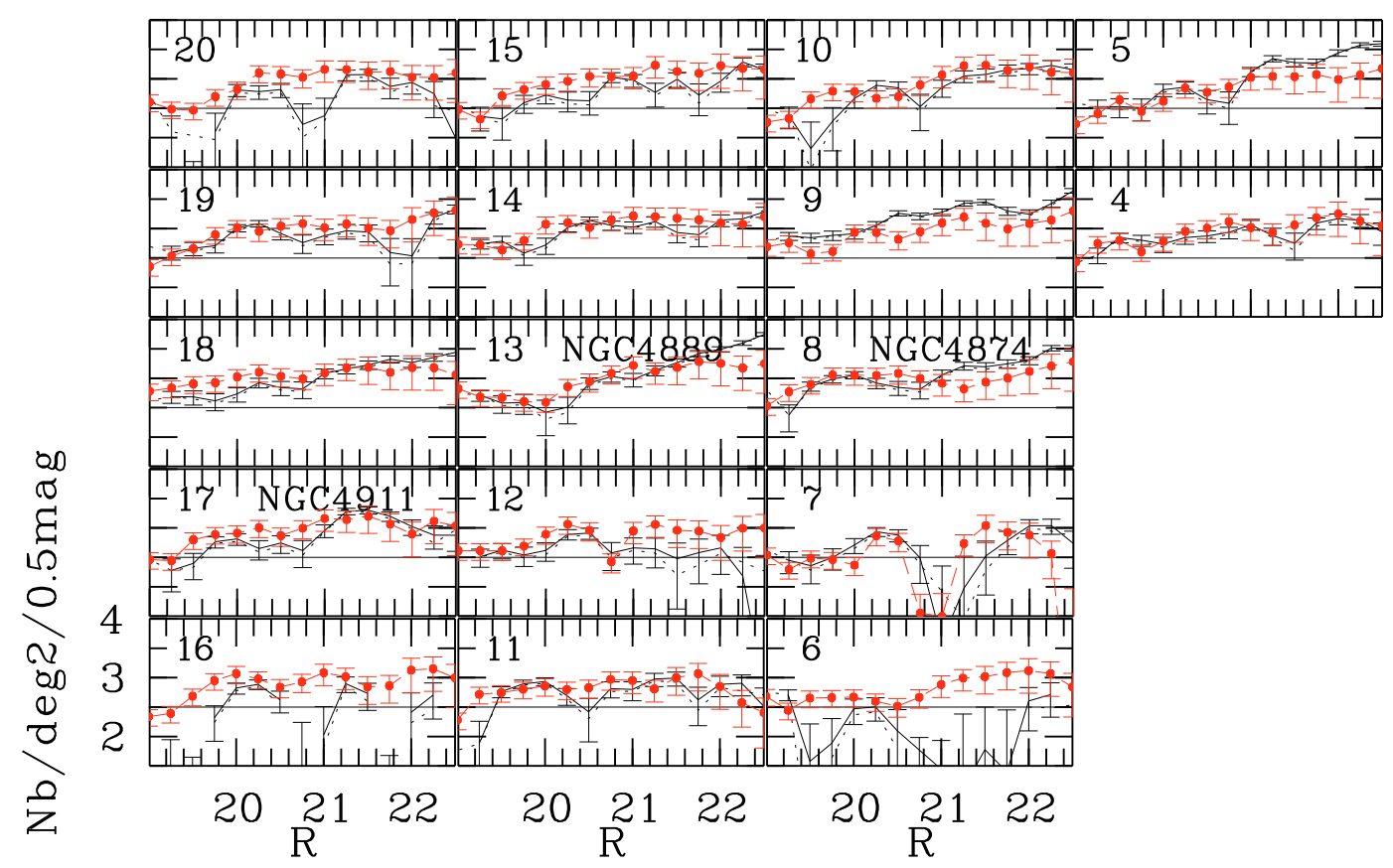

Fig. 8. $R$ band GLFs for different regions in the Coma cluster. North is top and east is left. The empty subgraphs correspond to areas where $B$ and $V$ data were not available. Black continuous and short dashed lines (along with error bars) are the GLF statistical estimates of Adami et al. (2007a). Red (grey in black and white version) long-dashed lines (along with error bars) are the present estimates.

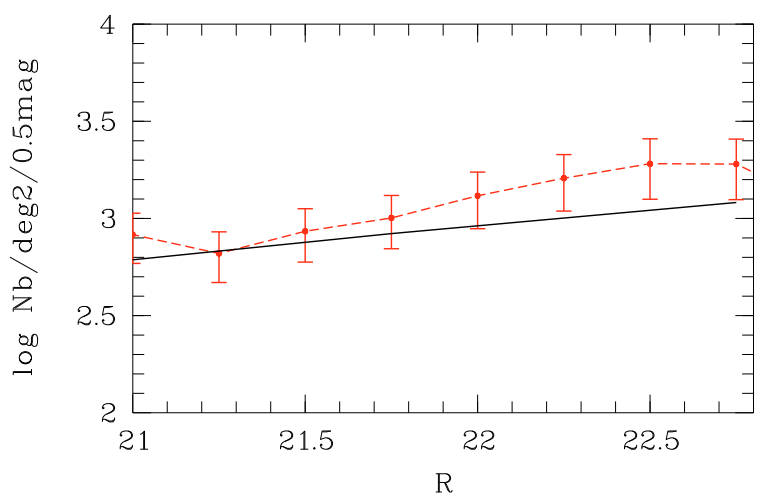

Fig. 9. $R$ band GLF for the NGC 4874 field computed with photometric redshifts (red dashed line - grey in black and white version). The slope from Secker et al. (1996) is overplotted (black full line).

\subsection{Galaxy luminosity functions in the $u^{*}$ band based on statistical background subtraction}

The Megacam $u^{*}$ band image is more extended than the CFH12K f.o.v. We typically have a 7 arcmin strip all around the CFH12K f.o.v. which is only covered in $u^{*}$. In order to compute $u^{*}$ GLFs in this external area, we applied the same statistical field galaxy subtraction technique as in Adami et al. (2007a and $b$ ). The main goal of this computation was to investigate potential variations of the GLF faint end slope in the $u^{*}$ band, which is sensitive to recent star formation bursts, and to compare our results to those of Donas et al. (1995).

The comparison fields were those among the deep CFHTLS fields (D1, D3, and D4) that are deep enough to allow us to make a statistical subtraction from our Coma data. In order to limit these catalogs to the same depth, we selected only galaxies brighter than $u *=24$ in total magnitude and brighter than 26.3 in surface brightness.

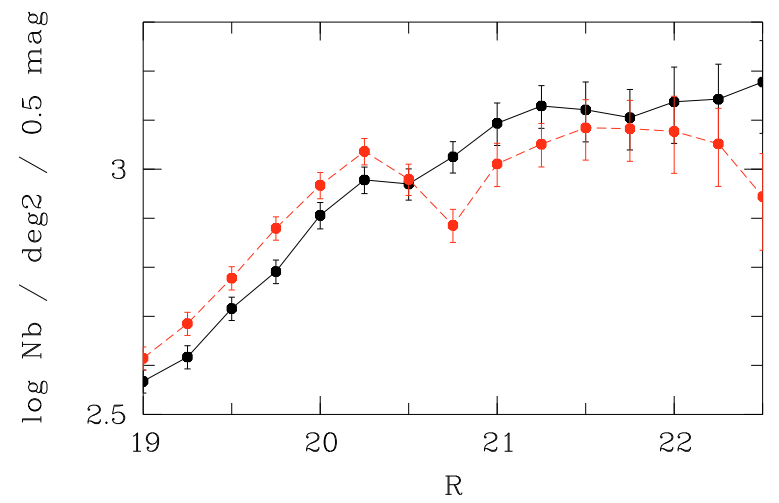

Fig. 10. $R$ band GLF for the northern (black) and southern (red dashed line - grey in black and white version) parts as defined in Adami et al. (2007a).

The comparison fields cover a total area of more than $3 \mathrm{deg}^{2}$, thus reducing the cosmic variance (see Adami et al. 2007a). The resulting GLFs are similar to the $u^{*}$ band GLFs computed in the CFH12K f.o.v. based on photometric redshifts. We sub-divided the Megacam f.o.v. in $7 \times 6$ subfields and computed the slope $\alpha$ of the $u^{*}$ GLF (computed applying a statistical subtraction and modelled by a Schechter function) in the $u^{*}=[22,24]$ mag range for each zone. The $42 \alpha$ slopes are given in Table 3 and were used to generate Fig. 11, where the contours of GLF slope $\alpha$ within the Megacam f.o.v. are displayed.

We clearly see a steepening of the $u^{*}$ GLF slope between the center and outskirts, changing from -1 to -2.2 . The regions with the steepest $u^{*}$ GLFs correspond to those where X-ray subtructures are detected and are located at $\sim 25^{\prime}$ from the cluster center. This is exactly where Donas et al. (1995) detected an enhancement in the median UV flux and in the fraction of bright blue star forming galaxies considered as cluster members. 
Table 3. Slope (and associated 1- $\sigma$ uncertainty) of the $u^{*}$ Schechter luminosity function between $u^{*}=22$ and 24 as a function of coordinates. These values were used to generate Fig. 11.

\begin{tabular}{llll}
\hline \hline$\alpha$ & $\delta$ & Slope & Err. slope \\
\hline 12.9639 & 27.5911 & -1.36 & 0.22 \\
12.9639 & 27.7339 & -2.22 & 0.61 \\
12.9639 & 27.8767 & -1.52 & 0.18 \\
12.9639 & 28.0194 & -1.59 & 0.28 \\
12.9639 & 28.1622 & -1.18 & 0.17 \\
12.9639 & 28.3050 & -1.96 & 0.21 \\
12.9639 & 28.4478 & -2.20 & 0.27 \\
12.9761 & 27.5911 & -1.48 & 0.20 \\
12.9761 & 27.7339 & -1.71 & 0.21 \\
12.9761 & 27.8767 & -1.61 & 0.09 \\
12.9761 & 28.0194 & -1.84 & 0.56 \\
12.9761 & 28.1622 & -1.84 & 0.56 \\
12.9761 & 28.3050 & -1.43 & 0.41 \\
12.9761 & 28.4478 & -1.96 & 0.40 \\
12.9883 & 27.5911 & -2.07 & 0.74 \\
12.9883 & 27.7339 & -0.34 & 0.57 \\
12.9883 & 27.8767 & -1.67 & 0.31 \\
12.9883 & 28.0194 & -0.13 & 0.65 \\
12.9883 & 28.1622 & -0.98 & 0.28 \\
12.9883 & 28.3050 & -1.81 & 0.23 \\
12.9883 & 28.4478 & -0.08 & 1.51 \\
13.0006 & 27.5911 & -1.41 & 0.49 \\
13.0006 & 27.7339 & -0.32 & 0.63 \\
13.0006 & 27.8767 & -1.66 & 0.33 \\
13.0006 & 28.0194 & -1.62 & 0.24 \\
13.0006 & 28.1622 & -0.85 & 0.18 \\
13.0006 & 28.3050 & -1.08 & 0.26 \\
13.0006 & 28.4478 & -1.99 & 0.37 \\
13.0128 & 27.5911 & -0.73 & 0.16 \\
13.0128 & 27.7339 & -1.18 & 0.73 \\
13.0128 & 27.8767 & -1.25 & 0.22 \\
13.0128 & 28.0194 & -1.06 & 0.13 \\
13.0128 & 28.1622 & -1.96 & 0.41 \\
13.0128 & 28.3050 & -1.51 & 0.18 \\
13.0128 & 28.4478 & -2.62 & 0.53 \\
13.0250 & 27.5911 & -1.88 & 0.37 \\
13.0250 & 27.7339 & -1.52 & 0.39 \\
13.0250 & 27.8767 & -2.12 & 0.78 \\
13.0250 & 28.0194 & -2.30 & 0.43 \\
13.0250 & 28.1622 & -2.12 & 0.28 \\
13.0250 & 28.3050 & -1.43 & 0.18 \\
13.0250 & 28.4478 & -1.63 & 0.36 \\
\hline & & &
\end{tabular}

\section{Multicolor type spatial distribution}

The goal of this section is to investigate the spatial distribution of galaxies according to their multicolor type. We stress here once again that our multicolor types are based on a color classification in a 5 mag space and are not real morphological types. Real morphological types are already not well determined at $R \geq 18$ (our starting magnitude) and completely unknown at $R \geq 22$, waiting for the results of the Coma cluster HST imaging survey (e.g. Carter et al. 2008). If we consider the morphological types compiled in Biviano et al. (1996) for 8 galaxies fainter than $R=18$ for which we computed photometric redshifts, we find that all are classified as elliptical galaxies and all have a multicolor type assigned to Delta bursts or elliptical galaxies, from the Bruzual \& Charlot (2003) evolutionary synthetic SEDs. This agreement clearly needs, however, to be confirmed.

Due to masked regions, we postpone the galaxy density profile per multicolor type determination to a future work and limit our analysis to the search of galaxy concentrations outside the masked areas.

In order to estimate the multicolor type spatial distribution inside the Coma cluster, we must adress the problem of $z \leq 0.2$ non Coma member galaxies (field or loose group objects) that cannot be discriminated by the photometric redshift technique alone.

If we consider the field galaxy luminosity function computed by Ilbert et al. (2006b) from similar data, we find that the field contribution represents about $15 \%$ of the Coma cluster galaxies down to $R=24$. Among these $15 \%$, about $1 / 4$ th are bulge galaxies and 3/4 are disk galaxies. However, this contribution is spread over the whole field of view and will act as a homogeneous background contribution of galaxies. It will therefore not modify the relative variation of the estimated multicolor types inside the Coma cluster. We could argue that dense regions of filaments (without being real massive structures such as clusters or groups) at $z \leq 0.2$ could provide different galaxy type counts across the field of view because they probably contain more early type galaxies. However, even considering the largest known cosmic bubble sizes, at least a dozen of such bubbles are superimposed between Coma and $z=0.2$. The sum of all these bubbles therefore homogenizes the distribution of field galaxy morphological types at $z \leq 0.2$.

Similarly, we estimated that the contribution of galaxies in loose groups represents $\sim 5 \%$ of the Coma cluster galaxies down to $R=24$ over the whole field of view. However, these contributions are concentrated in precise locations, so locally, the contribution can be much higher. Assuming the group GLF of Zandivarez et al. (2006) and the galaxy spectral type estimates in low mass groups $\left(\leq 10^{13.5} M_{\odot}\right)$ of Dominguez et al. (2002), we estimate that along the lines of sight to loose groups, galaxies in groups can represent more than $85 \%$ of all $z \leq 0.2$ galaxies. This contribution is on the order of $50 \%$ for early types galaxies and can reach $100 \%$ for other galaxy types. As we cannot estimate precisely the galaxy type contributions for the 5 loose groups detected behind Coma, this clearly suggests that we have to remove these areas.

Maps of galaxy multicolor type density were then generated from a simple count in cell technique, avoiding the masked regions and considering the whole sample of galaxies down $R=24$. The original cell size was $1^{\prime}$ and we applied an additionnal smoothing of $3 \times 3$ pixels in Fig. 12 . We only took into account cells completely included in our field of view, so there are no edge effects. This figure shows that early type galaxies are spread over the whole field of view with the possible exception of the north-west regions. Early and late Spirals show localised density enhancements in the cluster outskirts. We note however that the late (and perhaps early) spiral concentration located north of the cluster center could be well explained by the loose G4 group. Later types (irregulars and starbursts) are also distributed preferentially in the cluster outskirts. In particular, starburst galaxies are mainly located at the northwest, where X-ray substructures are also detected as an infalling group (see Adami et al. 2005a) and where a concentration of early multicolor types is also detected. This is also the place where the $u^{*}$ GLF is the steepest of the field (see previous section).

The first thing to note in Fig. 12 is that we confirm that the Coma cluster is mainly populated by early type galaxies $(\sim \mathrm{E}+\mathrm{S} 0)$, as expected for such a rich structure: about $80 \%$ of the galaxy population is made of ellipticals. Spirals represent only $15 \%$ of the faint galaxies.

Second, as expected and observed for bright galaxies (e.g. Whitmore et al. 1993), there are later type galaxy clumps in 

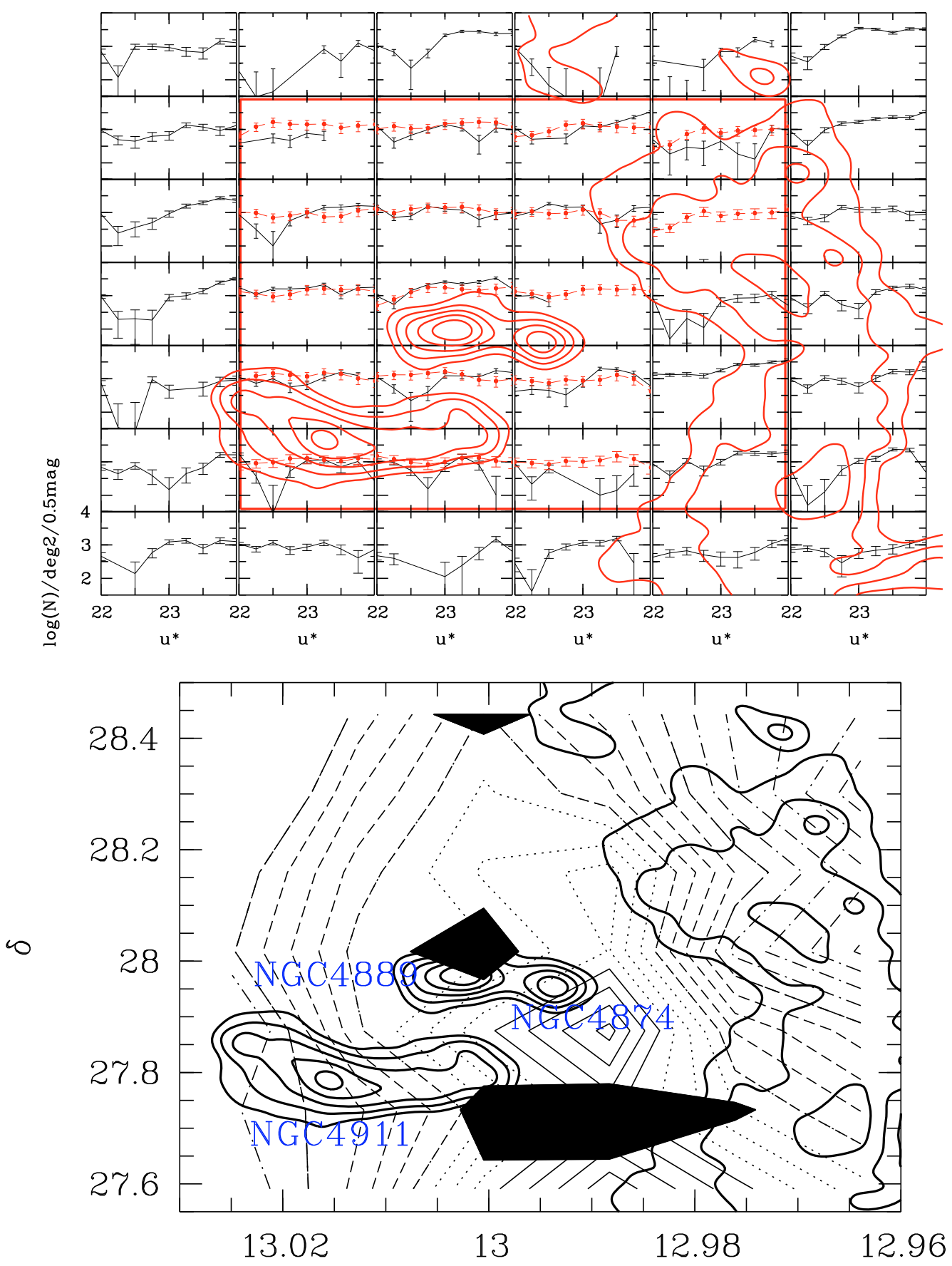

$\alpha$

Fig. 11. Upper panel: $u^{*}$ band GLFs for different regions in the Coma cluster computed with a statistical subtraction (continuous black lines with error bars) and the photometric redshift technique (red (grey in black and white version) dots linked with dashed lines and error bars). North is top and east is left. The CFH12K field of view is shown as the central red (grey in black and white version) rectangle. The empty subgraphs correspond to areas where the field counts were greater than the cluster counts. X-ray substructures are shown as thick continuous contours. Lower panel: contours of the GLF slope within the Megacam f.o.v. (thin continuous lines: slopes between -1 and -1.2 , thin dotted lines: between -1.2 and -1.4 , thin long dashed lines: between -1.4 and -1.6 , thin long dot-dashed lines: between -1.6 and -2.2 ). Shaded areas correspond to zones where the slope is not significantly different from 0 at more than a $2 \sigma$ level. X-ray substructures from Neumann et al. (2003) are shown as smooth thick black contours.

the cluster outskirts. This behavior essentially known for bright galaxies ( $R \leq \sim 18$ for the Coma cluster) is now extended 6 mag deeper, and for a finer and more objective type separation.
Third, we also see a clear concentration of starburst objects in a place where early spiral multicolor types are also detected and where X-ray substructures are present. These starburst 


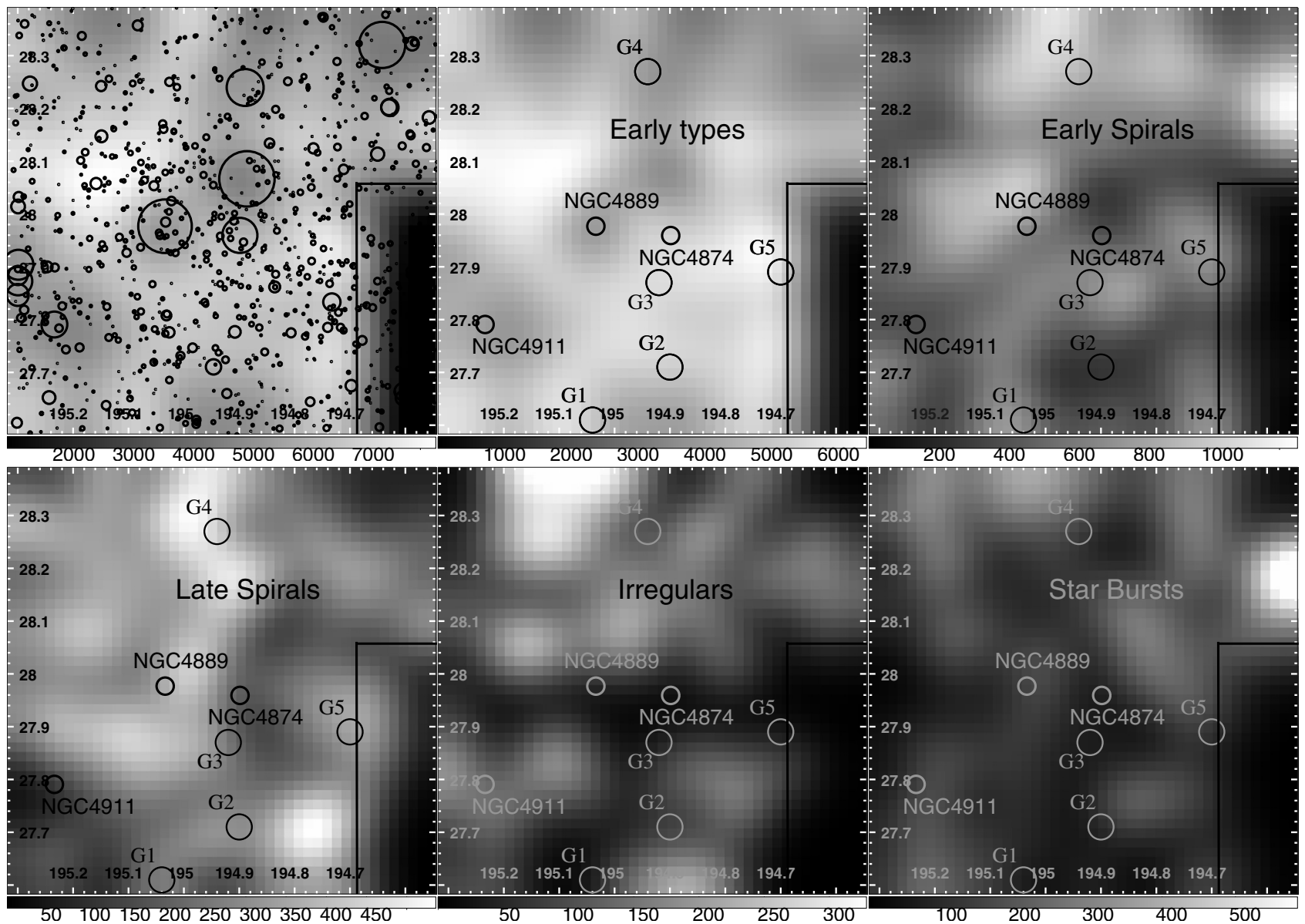

Fig. 12. Density contours of the various multicolor galaxy types: upper left: all multicolor types overplotted with masked regions (circles), upper middle: early type galaxies (types 1), upper right: early spirals (types 2), lower left: late spirals (types 3), lower middle: irregulars (type 4), lower right: starburst galaxies (type 5). The lighter color in the maps, the highest the number density peak. In each figure, north is up and east is left, the total field of view is that of the CFH12K $\left(42 \times 52 \mathrm{arcmin}^{2}\right)$, and the greyscale levels are given in number of galaxies per deg${ }^{2}$. The $5 \mathrm{detected}$ loose groups are indicated as large circles of $300 \mathrm{kpc}$ diameter (at $z=0.1$ ). Small circles give the positions of the three brightest galaxies in the field. In each of the panels, we finally show the south-west area where $B$ and $V$ filters are missing.

galaxies could see their star formation rate increased by interaction with the intra cluster medium.

\section{Conclusions}

We have shown that we can reproduce the GLFs computed by Adami et al. (2007a) by selecting cluster galaxies with PDFs from photometric redshift techniques. This both puts the Adami et al. (2007a) conclusions on a firmer ground, in terms of infalling directions and processes acting on galaxies inside the cluster, and proves that the photometric redshift technique (with a judicious cut in redshift) can help to select cluster members in nearby structures.

We clearly show that the $u^{*}$ GLF is steeper in the cluster outskirts, as already suggested by Donas et al. (1995). This could be due to a short burst of star formation in the faint galaxies, induced by the pressure of the intracluster medium (see also e.g. Cortese et al. 2007; or Boué et al. 2008).

We plan in a future paper to investigate how GLF and general galaxy properties (based on photometric redshift estimates) vary with the local galaxy density as derived for bright galaxies by Dressler et al. (1980).
Acknowledgements. The authors thank the referee for many useful and constructive comments.

We are grateful to the CFHT and Terapix (for the use of QFITS, SCAMP and SWARP) teams, and to the French CNRS/PNG for financial support. M.P.U. also acknowledges support from NASA Illinois space grant NGT5-40073 and from Northwestern University. The authors thank J. Secker, P. Côté and J. B. Oke for providing us with Keck spectra.

\section{References}

Adami, C., Biviano, A., \& Mazure, A. 1998, A\&A, 331, 439

Adami, C., Ulmer, M. P., Durret, F., et al. 2000, A\&A, 353, 930

Adami, C., Biviano, A., Durret, F., \& Mazure, A. 2005a, A\&A, 443, 17

Adami, C., Slezak, E.., Durret, F., et al. 2005b, A\&A, 429, 39

Adami, C., Picat, J. P., Savine, C., et al. 2006a, A\&A, 451, 1159

Adami, C., Scheidegger, R., Ulmer, M. P., et al. 2006b, A\&A, 459, 679

Adami, C., Durret, F., Mazure, A., et al. 2007a, A\&A, 462, 411

Adami, C., Picat, J. P., Durret, F., et al. 2007b, A\&A, 472, 749

Adami, C., Pelló, R., Ulmer, M. P., et al. 2008, A\&A, submitted

Bertin, E., \& Arnouts, S. 1996, A\&AS, 117, 393

Biviano, A., Durret, F., Gerbal, D., et al. 1996, A\&A, 311, 95

Bolzonella, M., Miralles, J.-M., \& Pelló, R. 2000, A\&A, 363, 476

Boué, G., Adami, C., Durret, F., Mamon, G., \& Cayatte, V. 2008, A\&A, 497, 335

Bournaud, F., Duc, P. A., \& Masset, F. 2003, A\&A, 411, L469

Bruzual, G., \& Charlot, S. 2003, MNRAS, 344, 1000 
Carter, D., Goudfrooij, P., \& Mobasher, B. 2008, ApJS, 176, 424

Coleman, G. D., Wu, C.-C., \& Weedman, D. W. 1980, ApJS, 43, 393

Cortese, L., Marcillac, D., Richard, J., et al. 2007, MNRAS, 376, 157

Dominguez, M. J., Zandivarez, A. A., Martinez, H. J., et al. 2002, MNRAS, 335 825

Donas, J., Milliard, B., \& Laget, M. 1995, A\&A, 303, 661

Dressler, A. 1980, ApJ, 236, 351

Fukugita, M., Shimasaku, K., \& Ichikawa, T. 1995, PASP, 107, 945

Ilbert, O., Tresse, L., Zucca, E., et al. 2005, A\&A, 439, 863

Ilbert, O., Arnouts, S., McCracken, H. J., et al. 2006a, A\&A, 457, 841

Ilbert, O., Lauger, S., Tresse, L., et al. 2006b, A\&A, 453, 809

Kinney, A. L., Calzetti, D., Bohlin, R. C., et al. 1996, ApJ, 467, 38

Kron, R. G. 1980, ApJS, 43, 305

McCracken, H. J., Ilbert, O., Mellier, Y., et al. 2008, A\&A, 479, 32
Meneux, B., Le Fèvre, O., Guzzo, L., et al. 2006, A\&A, 452, 387

Neumann, D. M., Lumb, D. H., Pratt, G. W., \& Briel, U. G. 2003, A\&A, 400, 811

Pickles, A. J. 1998, PASP, 110, 863

Polletta, M., Tajer, M., Maraschi, L., et al. 2007, ApJ, 663, 81

Secker, J., \& Harris, W. E. 1996, ApJ, 469, 623

Secker, J., Harris, W. E., Cote, P., \& Oke, J. B. 1998, in Proceedings of A New Vision of an Old Cluster: Untangling Coma Berenices, ed. A. Mazure, F. Casoli, F. Durret, \& D. Gerbal (World Scientific Publishing Co. Pte. Ltd.), 115

Serna, A., \& Gerbal, D. 1996, A\&A, 309, 65

White, S. D. M., Clowe, D. I., Simard, L., et al. 2005, A\&A, 444, 365

Whitmore, B. C., Gilmore, D. M., \& Jones, C. 1993, ApJ, 407, 489

Zandivarez, A., Martinez, H. J., \& Merchan, M. E. 2006, ApJ, 650, 137 